\title{
Toxicological pitfalls in ICU practice
}

\author{
Tomasz Janus ${ }^{1}$, Krzysztof Pabisiak ${ }^{2}$ \\ ${ }^{1}$ Department of Clinical and Forensic Toxicology, Pomeranian Medical University, Szczecin, Poland \\ ${ }^{2}$ Department of Nephrology Transplantology and Internal Medicine, Szczecin, Poland \\ Contributed equally
}

\begin{abstract}
Either analgosedation or central nervous system dysfunction may be a side effect of implemented pharmacological treatment, as well as a consequence of intentional or unintentional poisoning. In traumatic lesions or anoxia of the central nervous system, a question arises after a recommended follow-up period about the effects of xenobiotics on nervous system function. Although therapeutic drug monitoring is the gold standard in such cases, usually a single toxicological estimation of "a neurodepressive compound" is performed after treatment discontinuation in order to determine the type and amount of exogenous substances, or their metabolites, in a patient's bodily fluids, which allows for an assessment of its actual effects on central nervous system functions. The aim of this paper was to describe the aspects of diagnostic toxicology which are essential for improved determination of the type and amount of exogenous substances present in biological fluids of intensive care patients. We present examples of clinical cases in order to discuss the most common discrepancies in interpretation related to the ordering of toxicology tests.
\end{abstract}

Anaesthesiology Intensive Therapy 2018, vol. 50, no 5, 378-383

Key words: xenobiotics, neurodepressants; toxicology, diagnostics; ICU, treatment; drugs, therapeutic monitoring

Medications commonly used in inpatient treatment belong to a well-known group of compounds. Nevertheless, their pharmacological profiles were determined under homeostatic conditions. However, the body systems functioning as a whole in a patient requiring intensive care support are significantly deteriorated, which has an important impact on the pharmacokinetic and pharmacodynamic parameters of xenobiotics, which can be different from those observed in the reference status [1]. The pharmacokinetic parameters of exogenous substances, such as distribution volume and elimination, in a severely ill patient may significantly differ from the state in which they were defined. The biological half time, which is essential for estimating the potency of a drug can only serve as an indicative basis for estimating the elimination rate. The course of xenobiotic elimination in a patient with significantly disturbed homeostasis due to e.g. head trauma, cardiac arrest or haemorrhagic stroke is unknown. Neuroprotective analgosedation is the most common therapeutic approach in such cases. This is a generally accepted therapeutic management strategy despite the lack of a proven therapeutic benefit documented in clinical trials [2]. In the case of benzodiazepines, data regarding their impact on the central nervous system (CNS) can be drawn from studies on animal models [3-5]. From the pharmacological and toxicological point of view, it is necessary to determine the baseline levels of a xenobiotic, as well as those immediately after therapy discontinuation, in order to estimate the course of variation in xenobiotic concentration over time, which is not a routine procedure. In this type of management, it is extremely difficult, if not impossible, to detect a decrease in drug concentration to clinically insignificant values, and thus confirm that the therapeutic effect has been resolved based only on the pharmacokinetic data from the specification of the medicinal product.

\section{WHY A CLINIC ASKS FOR TOXICOLOGY DATA}

If it is not possible to obtain objective and reliable medical history in a patient with suspected intoxication, an inquiry into CNS depression based on a toxicological differential diagnosis with an unspecified aetiology should 
be performed. It is worth noting that such an inquiry should take into account hundreds of chemical compounds in different chemical states and with diverse chemical structures. The number of compounds that may be the actual cause of nervous system dysfunction, and therefore the number of parameters to be determined, significantly exceeds the number of parameters analysed in any other field of medical diagnostics. Procedures used in general diagnostic toxicology should allow for the detection of such compounds as alcohols and aliphatic glycols, volatile organic solvents, medications from different therapeutics groups, designer psychoactive substances, narcotics or pesticides. The designer drug list alone includes over 400 substances with diverse structures, which renders their identification a challenge even for an adequately equipped toxicology laboratory [6]. To approach this issue, it is necessary to answer a clinical question on whether we are dealing with a xenobiotic and its therapeutic, toxic or lethal effects. The availability and selection of an appropriate analytical tool is of key importance for the correct interpretation of results and the precise diagnosis of poisoning.

\section{WHAT A CLINIC CAN EXPECT FROM TOXICOLOGY DATA}

The multitude of probable chemical compounds to consider and evaluate does not allow for a completely undirected toxicological investigation. Although this type of diagnosis uses different analytical techniques, cooperation with the doctor in charge is essential. Information on the timing and circumstances of intoxication, along with the clinical picture and laboratory findings regarding the vital organs involved in drug metabolism may be helpful and sometimes essential. This data allows for the selection of optimal analytical techniques in order to identify exogenous substances. Unfortunately, in everyday practice, a doctor's order for a toxicological assessment often lacks even basic information, such as patient data and the circumstances of poisoning, with "toxicological profile" being the only annotation. The lack of data that could provide some guidance often prolongs the diagnostic process, increases its costs, or even prevents eventual identification of the xenobiotic causing the intoxication. Among the current methods used in routine toxicological investigation, it is important to note two techniques: immunological assays and instrumental methods. Intoxications with known or suspected aetiology can be successfully verified using immunological methods. Although these methods are mainly intended to assess the urinary levels of xenobiotics, they may be also used, in some cases, to verify a patient's blood. Immunological assay kits are widely available and relatively easy to use. The measurement is usually performed directly on biological material (without the need to additionally process the sample) with the result being available within
15 to 20 minutes. On the other hand, immunological assays have numerous limitations [7]. First of all, they are characterised by an unsatisfactory substrate specificity leading to false positive results. Additionally, the range of testable parameters is limited and there is no possibility of general (non-targeted) diagnosis. In practice, these methods are useful in confirming intoxication with selected xenobiotics, such as acetaminophen, carbamazepine, theophylline, salicylates, valproic acid, digitoxin and phenobarbital. Such tests are also used to identify the main groups of narcotics such as amphetamines, methamphetamine, 3,4-methylenedioxymethamphetamine (ecstasy), opiates, tetrahydrocannabinols and cocaine. Despite the availability of immunologic assays for other xenobiotics such as benzodiazepines, tricyclic antidepressants and opiates, the results of such test may be inconclusive and lead to a false diagnosis [8]. Therefore, instrumental methods are necessary in most cases in order to obtain accurate results from qualitative and quantitative toxicology. Instrumental methods are used for all types of investigated material (blood, urine, gastric lavage sample) and are based on chromatographic separation and the direct detection of a xenobiotic. Considering the wide range of available tests, it is necessary to use several analytical systems based on different detection methods and separation techniques (there are no universal solutions). Intoxication diagnosis after the ingestion of an unknown alcohol involves chromatographic analysis with flame-ionisation detection with separation in different vehicles (different chromatographic columns), using specific equipment settings for glycols, aliphatic alcohols and volatile organic solvents. Gas chromatography-mass spectrometry, usually at low resolution in a single or tandem setup, is used for the assessment of intoxication with less volatile, non-polar organic compounds (pesticides, dioxins, polychlorinated biphenyls). Fluid chromatography with photodiode detection is used to identify more polar compounds (mainly drugs). Despite high efficacy in the identification of a number of compounds, the use of instrumental methods is limited by insufficiently specific UV spectra and relatively low specificity (mostly for narcotics or designer psychoactive compounds, or drugs with potent pharmacological activity). Such cases require the use of high-resolution tandem mass spectrometry, which allows for a simultaneous quantitative and qualitative identification of hundreds of organic compounds present at very low levels. In a general toxicological analysis, sample processing before analysis is also important and depends to a large extent on the structure of the analysed compounds. For example, confirmation of intoxication with baclofen or metformin (both highly polar compounds soluble in water) requires a specific procedure of extraction which will allow for the selective transition of these drugs to a solution intended for analysis. 


\section{A THERAPEUTIC, TOXIC OR LETHAL DOSE?}

The lack of a basic unified terminology in the crosstalk between clinicians and toxicological personnel leads to confusion, with the frequently used, yet not clearly specified term "screening test". The term is usually narrowed down to define a group of xenobiotics: medications, narcotics or alcohols not for human consumption and designer drugs. Unfortunately, this term is understood differently by different people involved in the diagnostic process (laboratory technicians, doctors, toxicologists). Moreover, a screening test is often incorrectly defined as an immunological assay or strip/dipstick test rather than the actual range of analysed parameters and providing at least a theoretical possibility of identifying a group of xenobiotics in a single analytical cycle. The latter option would be helpful for a referring physician whose role is to either confirm or exclude the presence of substances that could have an impact on the patient's CNS function, as opposed to compounds that can be detected only by the immunological method. In the diagnostic reality, answering a clinician's question about the type of intoxication present is possible only after a multifactorial analysis. Only instrumental methods can confirm the presence of, among other things, diabetic medications, calcium channel blockers, beta blockers, phenothiazines, NSAIDs or antihistamines. Immunological tests cannot be a basis for deduction, especially in the context of their effects on the CNS. It is also important to note that different agents from the same therapeutic group show different reactivity in immunological assays. Table 1 shows exemplary detection levels for benzodiazepines in immunological tests compared with their therapeutic levels.

It is impossible to estimate the effects of benzodiazepines on the CNS using this assay as the limits of their detection are much higher than the upper ranges of therapeutic concentrations. Targeted testing, which is focused on a specific parameter, is another issue. Even in such cases it is not always recommended to use only immunological testing. For example, targeted testing to determine blood levels of midazolam and/or fentanyl, which would be impossible using only immunological assays as they can only detect large concentrations of midazolam and do not take into account its active metabolites, is often ordered by intensive care doctors. According to the data of the manufacturer of the widely used Emit tests (shown in Table 1), the minimal detection level for benzodiazepines is $300 \mathrm{ng} \mathrm{mL}^{-1}$. This method allows for detecting the majority of agents from this class only at concentrations far exceeding the therapeutic levels; therefore, it is difficult to consider it as a screening method - there is a possibility of a negative result despite the presence of the drug in the patient's blood at therapeutic levels.

\section{CLINICAL VIGNETTE}

The importance of choosing an appropriate analytical technique may be illustrated based on the case of toxicological analysis due to suspected zolpidem intoxication. The drug belongs to the imidazopyridines - a group of $G A B A_{A}$ receptor agonists with hypnotic properties. In everyday practice, this drug is assigned to the benzodiazepine group due to its hypnotic properties; however, as its chemical structure is completely different from that of other medications in this group, its detection using tests for benzodiazepines is impossible. In this particular case, the doctor ordered testing for tricyclic antidepressants (TCA) and benzodiazepines despite the fact that both of these tests would turn out to be negative even at very high levels of zolpidem. In clinical situations requiring targeted testing, it is advisable to avoid using group names, but use specific drug names instead. On the other hand, when ordering general screening, the range of suspected medication groups (alcohol, narcotics, medications) should be narrowed down. In many cases, providing the reason for toxicological testing may be of utmost importance and greatly advantageous for the whole diagnostic process.

In the case mentioned above, the physician on duty ordered immunological assays for benzodiazepines and opiates. The laboratory technician could have conducted the testing and send back negative results. However, after a telephone consultation with an intensive care doctor, it was found that midazolam and fentanyl were the drugs used. It is worth noting that the referring physician chose the term "opiates" in the test order despite the fact

Table 1. Benzodiazepines identification, therapeutic and toxic levels with reference to the EmitTox ${ }^{\mathrm{TM}}$ tests (cut-off at $=300 \mathrm{ng} \mathrm{mL} \mathrm{L}^{-1}$ ) [9]

\begin{tabular}{|c|c|c|c|c|c|c|c|}
\hline Substance & $\begin{array}{c}\text { Identification } \\
\text { range } \\
\left(\mathrm{ng} \mathrm{mL}^{-1}\right)\end{array}$ & $\begin{array}{c}\text { Therapeutic } \\
\text { range } \\
\left(\mathrm{ng} \mathrm{mL}^{-1}\right)\end{array}$ & $\begin{array}{c}\text { Toxic } \\
\text { range }\end{array}$ & Substance & $\begin{array}{l}\text { Identification } \\
\text { range } \\
\left.\text { (ng mL } L^{-1}\right)\end{array}$ & $\begin{array}{c}\text { Therapeutic } \\
\text { range } \\
\left(\mathrm{ng} \mathrm{mL}^{-1}\right)\end{array}$ & Toxic \\
\hline Alprazolam & 400 & $5-50$ & $100-400$ & Flumazenil & No data & $20-100$ & 500 \\
\hline Temazepam & 1,000 & $400-900$ & No data & Prazepam & 1,000 & $200-700$ & 1,000 \\
\hline Chlordiazepoxid & 5,000 & $400-3,000$ & $3,500-15,000$ & Flurazepam & No data & $20-100$ & $200-500$ \\
\hline Clonazepam & 2,000 & $2-80$ & 100 & Diazepam & 300 & $100-2,000$ & $3,000-5,000$ \\
\hline Lorazepam & 3,000 & $8-25$ & $300-500$ & Oxazepam & 1,000 & $200-1,500$ & 2,000 \\
\hline
\end{tabular}




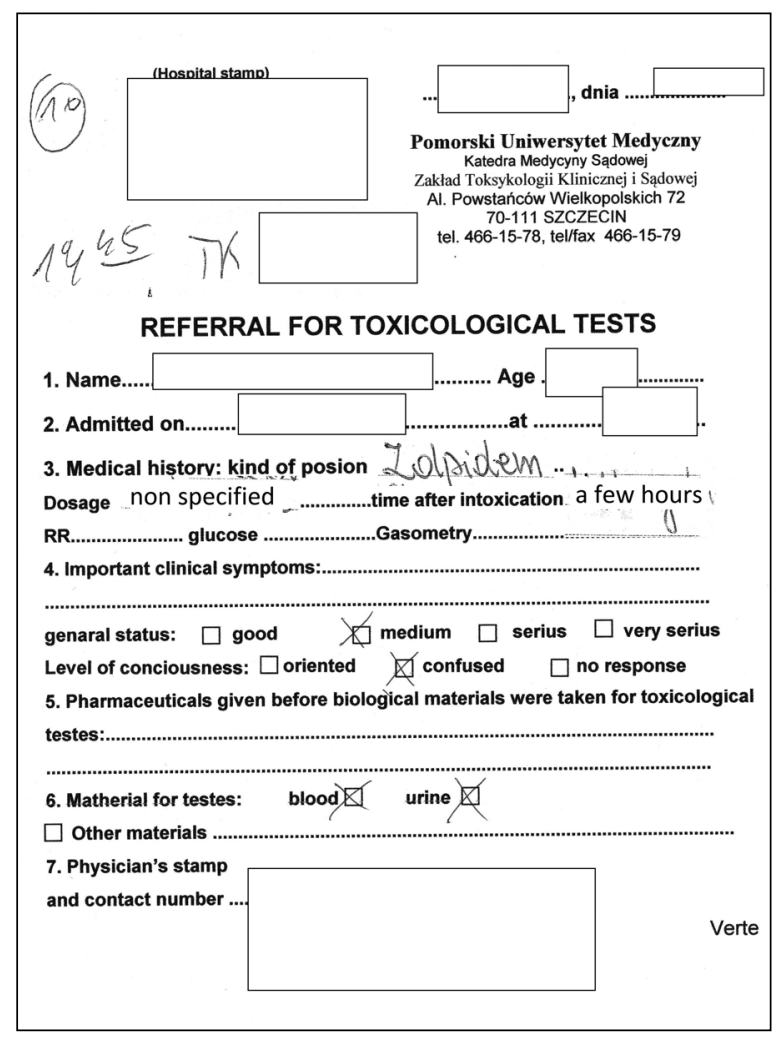

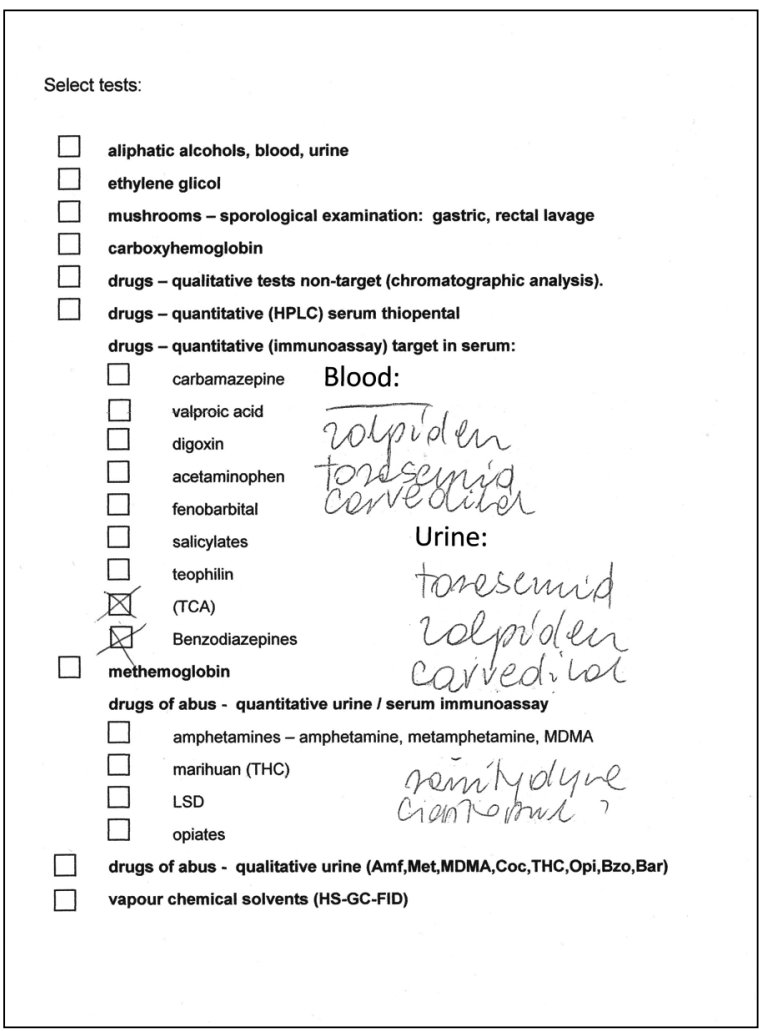

Figure 1. Referral for toxicology testing (at admission to hospital) in the case of a 64-year-old man with a suspected zolpidem overdose
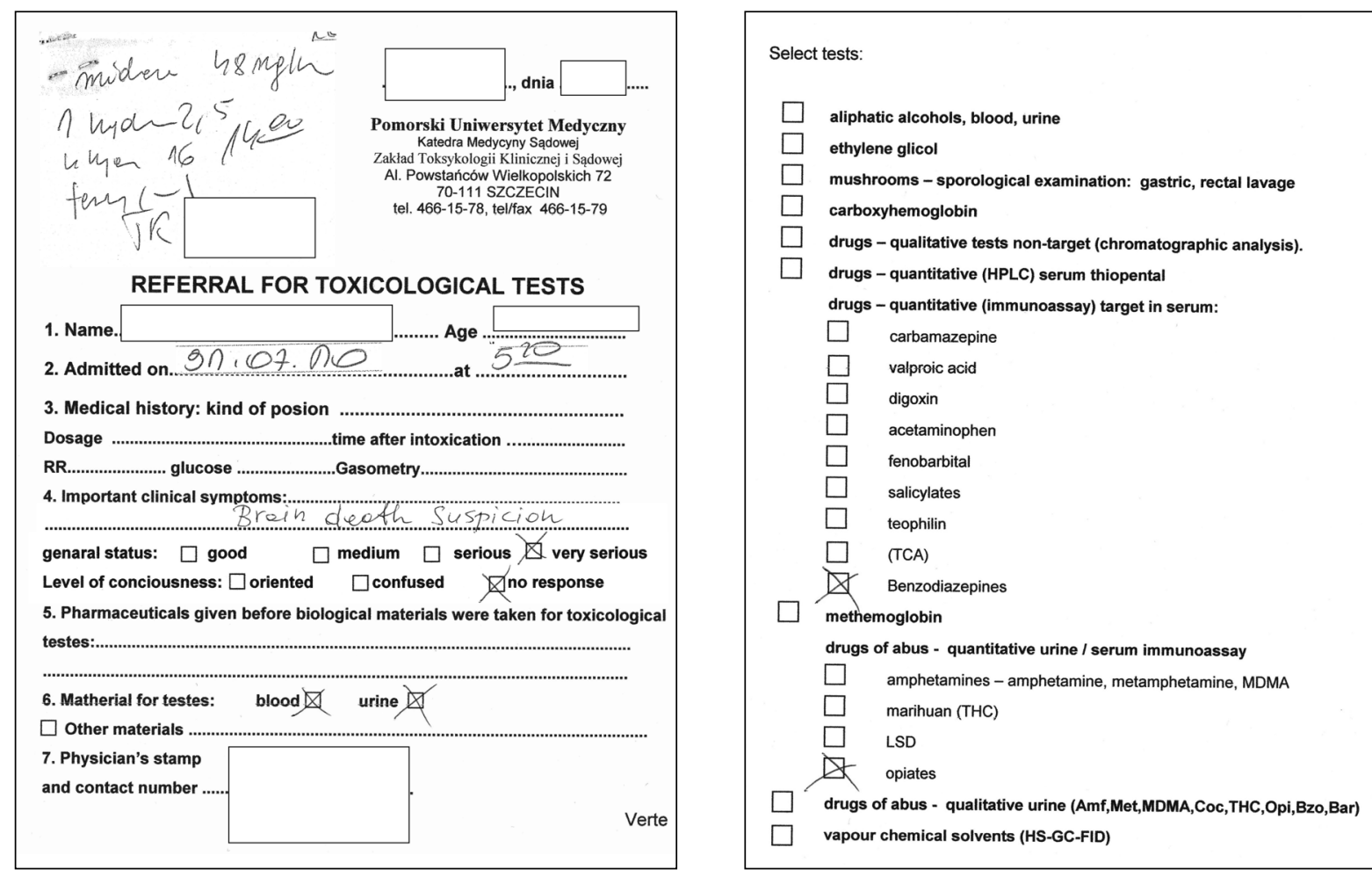

Figure 2. A 54-year-old male at 72-hours after admission to an ICU with a preliminary diagnosis of brain death 
Figure 3. Fentanyl, midazolam and active metabolites concentrations established by liquid chromatography quadrupole time-of-flight mass spectrometry (LC-Q TOF) for reference to Fig. 2 Therapeutic range for midazolam: $0.04-0.1 \mathrm{mg} \mathrm{L}^{-1}$

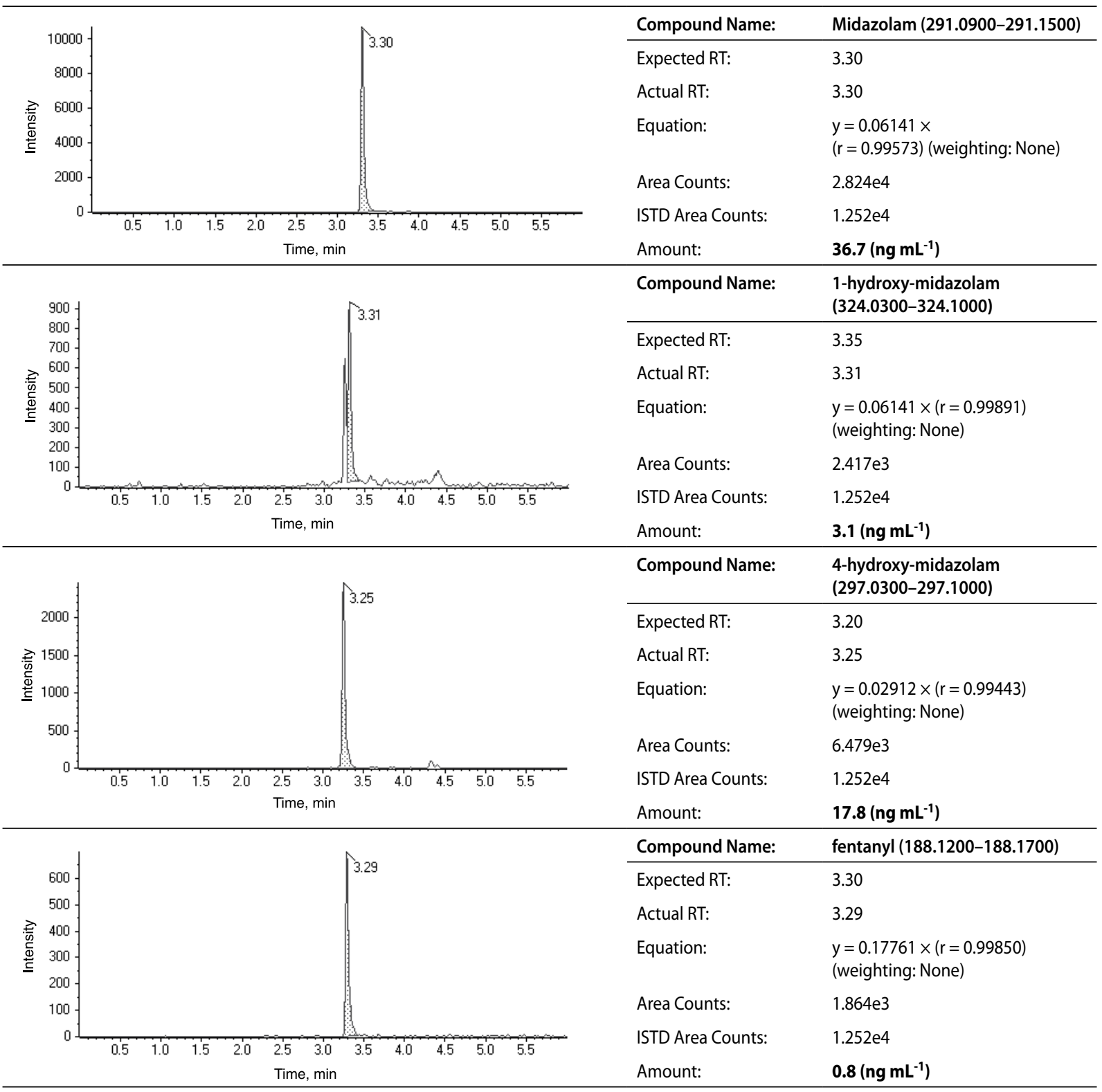

that fentanyl is a synthetic agonist at the opioid receptors. While ordering immunological test for benzodiazepines in cases of suspected midazolam intoxication may be justified by one's unfamiliarity with the specifics of immunological testing, referring to fentanyl as an opiate should be considered as a mistake. Measuring anaesthetic medication levels (fentanyl and midazolam along with their metabolites in particular) requires instrumental methods. The testing was conducted using liquid chromatography coupled with high resolution mass spectrometry, which allowed for the detection of medications used with expected sensitivity and selectivity. In cases where it is known that the tested xenobiotic is present at a low concentration, but still with a therapeutic or clinically significant effect, liquid chromatography with tandem mass spectrometry is the method of choice. In the above-presented case, the concentration of fentanyl was $0.8 \mathrm{ng} \mathrm{mL}^{-1}$ (the range of therapeutic concentrations: $3.0-300 \mathrm{ng} \mathrm{mL}^{-1}$ ).

\section{INSTRUMENTAL MEASUREMENTS IN TOXICOLOGY - "QUICK, PRECISE, INEXPENSIVE"}

Contrary to the commonly held view, targeted drug testing using high resolution mass spectrometry is not time consuming. For example, the measurement of selected drugs commonly used in an ICU setting (the so-called anaesthetic panel - fentanyl, midazolam and two of its 
metabolites, propofol and dexmedetomidine - takes no longer than 30 minutes (including sample preparation, analysis and result assessment). It should be added that the number of parameters analysed in one measurement cycle depends only on the ordering physician's suggestions. The simultaneous identification of a few to several compounds does not constitute a limitation of the method. However, it is important to specify all investigated compounds before method validation as the later inclusion of new parameters will mean the need to repeat the procedure. The latest instrumental methods allow one to reduce analysis duration, which is now comparable with that of rapid immunological assays, while retaining a higher selectivity, specificity, precision and range of parameters measured. For comparison, testing a single parameter using an immunological assay takes about 20 minutes, while a general testing due to drug intoxication using instrumental methods takes from 20 to 30 minutes. Measuring another compound with the immunological method requires another measurement cycle with a significantly limited range of measurable parameters. As opposed to immunological testing, where the cost of each single measurement is a multiplication of each unit cost, the increasing number of simultaneously tested parameters in instrumental methods is not associated with a proportional increase in the cost. In toxicological diagnostics, general analyses requiring a number of analytical devices and different procedures are the most expensive, while even in such cases the time for testing is usually not longer than two hours (especially when three different samples from the patient are used - blood, urine and gastric lavage fluid). Diagnostic toxicology for clinical purposes includes mainly two approaches - targeted determination of a selected xenobiotic and multiple parameter analysis (general testing), sometimes limited to one group of compounds (narcotics, alcohols). The choice of procedure used largely depends on the availability of data concerning a specific clinical case (past medical history of the patient, circumstances of intoxication, results of clinical and laboratory tests), which will in turn ensure the accuracy of the toxicological analysis. Two groups of methods are used in the diagnostic process. The use of immunological methods, which are easy and quick, is limited to testable parameters in targeted testing.

\section{SUMMARY}

Instrumental methods, which allow both for general (screening) and targeted analyses for compounds with high biological activity and which show high selectivity and appropriate accuracy, are of primary importance in the diagnosis of poisoning. In addition to having access to appropriate technical laboratory equipment, close cooperation between physicians and the laboratory team responsible for toxicological evaluation is an important element determining the appropriate level of diagnostic toxicology.

\section{ACKNOWLEDGEMENTS}

1. Source of funding: none.

2. Conflict of interest: none.

\section{References:}

1. Heinke W, Koelsch $\mathrm{S}$. The effects of anesthetics on brain activity and cognitive function. Curr Opin Anaesthesiol. 2005; 18(6): 625-631, doi: 10.1097/01.aco.0000189879.67092.12, indexed in Pubmed: 16534303.

2. Roberts DJ, Hall RI, Kramer AH, et al. Sedation for critically ill adults with severe traumatic brain injury: a systematic review of randomized controlled trials. Crit Care Med. 2011; 39(12): 2743-2751, doi: 10.1097/ CCM.0b013e318228236f, indexed in Pubmed: 22094498.

3. Saito T, Takeichi S, Osawa M, et al. Changes in brain barbiturate concentration in brain-dead rabbits. Nihon Hoigaku Zasshi. 1998; 52(3): 177-183, indexed in Pubmed: 9780661.

4. Saito T, Takeichi S, Tokunaga I, et al. Experimental studies on effects of barbiturate on electroencephalogram and auditory brain-stem responses. Nihon Hoigaku Zasshi. 1997; 51(5): 388-395, indexed in Pubmed: 9436367.

5. Wang W, Lu R, Feng DY, et al. Inhibition of microglial activation contributes to propofol-induced protection against post-cardiac arrest brain injury in rats. J Neurochem. 2015; 134(5): 892-903, doi: 10.1111/ jnc.13179, indexed in Pubmed: 26016627.

6. The list of NPSs identified by National Forensic Laboratory (NFL). http:// www.policija.si/apps/nfl response web/seznam.php (11.11.2016).

7. Ellefsen KN, Anizan S, Castaneto MS, et al. Validation of the only commercially available immunoassay for synthetic cathinones in urine: Randox Drugs of Abuse V Biochip Array Technology. Drug Test Anal. 2014 6(7-8): 728-738, doi: 10.1002/dta.1633, indexed in Pubmed: 24659527.

8. Spinelli E, Barnes AJ, Young S, et al. Performance characteristics of an ELISA screening assay for urinary synthetic cannabinoids. Drug Test Anal. 2015; 7(6): 467-474, doi: 10.1002/dta.1702, indexed in Pubmed: 25167963.

9. Schulz $M$, Iwersen-Bergmann $S$, Andresen $H$, et al. Therapeutic and toxic blood concentrations of nearly 1,000 drugs and other xenobiotics. Crit Care. 2012; 16(4): R136, doi: 10.1186/cc11441, indexed in Pubmed: 22835221.

\section{Corresponding author:}

\section{Krzysztof Pabisiak}

Department of Nephrology Transplntology and Internal Medicine

Powst. WIkp. 72, 70-111 Szczecin, Poland

bkpn@pum.edu.pl

Received: 13.05 .2018

Accepted: 21.11 .2018 